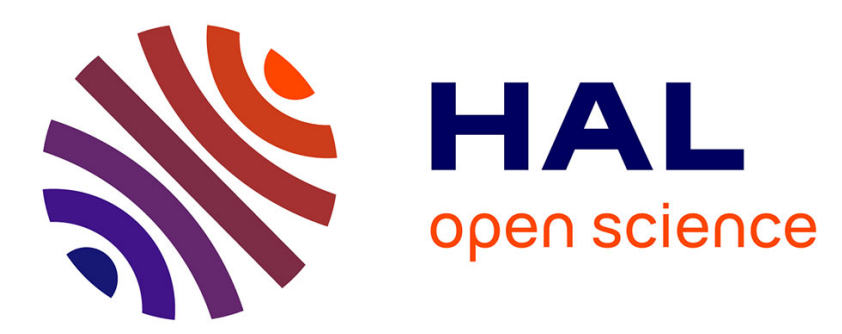

\title{
Nature des pâtes des céramiques culinaires des ateliers de la Sarra et de Trion
}

\author{
C. Batigne-Vallet, Anne Schmitt
}

\section{To cite this version:}

C. Batigne-Vallet, Anne Schmitt. Nature des pâtes des céramiques culinaires des ateliers de la Sarra et de Trion. Gallia - Archéologie de la France antique, 1997, Les productions des ateliers de potiers antiques de Lyon (2e partie) : les ateliers du Ier s. après J.-C., 54, pp.69-71. 10.3406/galia.1997.3238 . halshs-00980651

\section{HAL Id: halshs-00980651 https://shs.hal.science/halshs-00980651}

Submitted on 29 Jan 2020

HAL is a multi-disciplinary open access archive for the deposit and dissemination of scientific research documents, whether they are published or not. The documents may come from teaching and research institutions in France or abroad, or from public or private research centers.
L'archive ouverte pluridisciplinaire HAL, est destinée au dépôt et à la diffusion de documents scientifiques de niveau recherche, publiés ou non, émanant des établissements d'enseignement et de recherche français ou étrangers, des laboratoires publics ou privés.

\section{(ㅇ)(1) $\$$}

Distributed under a Creative Commons Attribution - NonCommercial - NoDerivatives| 4.0 


\title{
NATURE DES PÂTES DES CÉRAMIQUES CULINAIRES DES ATELIERS DE LA SARRA ET DE TRION
}

\author{
Cécile Batigne et Anne Schmitt
}

\section{ÉTUDE PÉTROGRAPHIQUE}

L'argile utilisée dans les deux atcliers de céramiques linaires lyonnais recensés à cc jour a pu être prélevéc sür place, c'est-à-dire sur la colline de Fourvic̀re. Il s'agit diit ne argile de type villafranchien déposée sur le plateau *a fin de l'ère tertiaire (Demarcq, 1973) et provenant 1. Inc altération du massif cristallin des Alpes. À cause de Lette origine détritique, elle contient beaucoup de fragments de granite, du quart\%, des feldspaths et des micas. lểe plus, elle est de nature non calcaire et de ce fait convient parfaitcment à la fabrication d'une céramique à vocation culinaire.

Nous nous sommes intéressées aux pâtes argileuses produites dans les deux ateliers à partir de cette argile. En observant l'abondance et la taille des inclusions, nous avons mis en évidence quatre sortes de pâtes :

- la pâte I contient des dégraissants de taille moyenne en abondance moyenne, et les éléments de grande taille, constitués essentiellement de fragments de granite, sont présents en faible quantité ;

- la pâte II présente le même type d'inclusions mais les gros grains et les fragments de roches sont plus abondants ;

- la pâte III montre peu de dégraissants visibles ;

- la pâte IV est abondamment dégraisséc d'inclusions calibrées de petite taille.

Les poticrs des deux ateliers n'ont pas utilisé ces quatre types de pâte de la même façon. Les objets façonnés avec les pâtes I et II sont majoritaires à la Sarra, alors que les pâtes III ct IV sont plus abondantes dans l'atelier de Trion (tabl. VI).

Les deux ateliers n'utilisaient donc pas les mêmes procédés pour préparer leur matière argileuse. Les potiers de la Sarra ne semblent pas avoir porté d'attention particulière à la préparation de leur pâte : la pâte I a dû être utiliséc telle qu'elle se présentait au moment de l'extraction. Ia pâte II devait provenir d'une partie du gisement où les fragments de granite et les micas étaient plus abondants, et ceux-ci n'ont pas tous été ôtés par le potier au moment de la préparation.

I es deux pâtes de l'atelier de Trion sont plus fines. Ia pâte III semble provenir d'une passée où l'argile est naturellement moins riche en éléments détritiques, et a dû être utilisće telle quelle. La pâte IV est probablement élaborée à partir de l'argile villafranchienne broyée et tamisée.

Les fouilles des ateliers nous apprennent que les potiers ont généralement utilisé indifféremment les deux pâtes argileuses qu'ils préparaient pour façonner tous les objets. L'exception à cette observation se situe dans le contexte de rebuts de cuisson mis au jour à la Sarra, où l'on constate que la pâte grossière II n'est employée que pour la réalisation de plats à cuire. Ceux-ci sont alors toujours de teinte rouge, c'est-à-dire qu'ils sont cuits en mode A. En revanche, lorsque l'on s'intéresse à la vaisselle culinaire utilisée sur les contextes d'habitat lyonnais, on perçoit quclques spécificités en ce qui concerne l'emploi des pâtes argilcuses, comme nous le verrons infra.

Pour revenir aux modes de cuisson, la céramique culinaire lyonnaise est majoritaircment cuite en atmosphère 
Tabl. VI. Tableau des pâtes des céramiques culinaires des ateliens de la Sarra et de Trion.

\begin{tabular}{|l|l|l|}
\hline & atelier de la Sarra & atelier de Trion \\
\hline pâte argileuse & plutôt grossière (l et II) & plutôt fine (III et IV) \\
\hline cuisson & $84 \%$ en mode B & $73 \%$ en mode B \\
\hline production & strictement culinaire & majoritairement culinaire \\
\hline
\end{tabular}

réductrice, c'est-à-dire en mode $B$, ce qui se traduit par des couleurs grises ou noires, et qui est conforme, semble-t-il, à une habitude autochtone gauloise. À l'atelier de la Sarra, on a certainement cuit la plus grande partie de la production dans des structures creusées dans le sol, comme il en a été attesté à la fouille. À Trion, les fouilleurs ont identifié un four à sole, mais rien ne nous permet d'affirmer que ce four ait été destiné à la cuisson des céramiques communes sombres, alors qu'il est plus probable qu'il a pu être utilisé pour la cuisson de la céramique commune claire du même atelier. Il est en effet plus aisé de faire une cuisson en atmosphère réductrice dans une fosse, alors qu'un four à sole est plus approprić à une cuisson en atmosphère oxydante.

I ces deux ateliers sont donc proches l'un de l'autre à plusicurs égards. Ils le sont géographiquement et l'on peut dire qu'ils font partie du meme site de production. I curs productions s’insèrent également dans les mêmes traditions technique et culinaire.

Néanmoins, si l'on souhaite les distinguer, on peut mettre en relation certaines caractéristiques des productions de chaque atelier à l'aide d'observations faites sur le site d'habitat de la rue des Farges, qui s'est approvisionné massivement en céramique culinaire lyomnaise; en ce qui concerne les objets faits à la Sarra, ils sont presque toujours gris ou noirs (dans $84 \%$ des cas) et ce sont des récipients majoritairement culinaires (pots à cuire, plats à cuire, marmites, bouilloires). A l'atelier de Trion, les vases gris oll sombres sont moins abondants $(73 \%)$, les pâtes sont moins dégraissées et les récipients qui sont façonnés avec ces pâtes ne sont pas toujours exclusivement culinaires. En effet, avec les pâtes III et IV, on a produit des jattes, des pichets et des ceramiques communes plus fines que la finition soignéce et la petite taille nous incitent à ne pas classer dans la batterie de vaissclle à feu (tabl. VI).

Enfin, sur le site de la rue des Farges, on a observé que de la vaisselle culinaire fabriquée avec ces quatre pâtes lyonnaises a été utilisée pendant toute la durée de l'occupation (de la fin du I" s. avant J.-C. au début du III's. apres J.-C.). Cela prouve que le site producteur de céramique culinaire de la colline a été en activité une dizaine d'anncés après l'installation de la colonic el jusqu'au début du III's. après J.-C:.

C. B.

\section{ANAI.YSES CHIMIQUES}

Ia pâte des céramiques communes sombres est constituéc d'une matrice argileuse contenant une fraction sableuse abondante et grossière, composéc essenticllement de grains de quartz, feldspaths, micas et fragments de roches granitiques. I a granulométrie de ces grains est continue, l'argile utilisée provient vraisemblablement d'un gisement dans lequel la fraction sableuse est naturellement abondante. I.es fluctuations d’abondance et de granulométrie de ce dégraissant naturel pourraient expliquer en partic les fluctuations de compositions chimiques. De plus, plusicurs textures de pâte ont été mises en évidence par les analyses macroscopiques.

Ia determination de la composition chimique des ceramiques communes sombres de l'atelier de la Sarra a écé réaliscé sur 25 exemplaires. I a pâte appartenant au type I étant la micux représentée sur le site de la Sarra, la majorité des exemplaires analysés appartiennent à ce groupe. Six exemplaires se sont avérés avoir des compositions très marginales et n’ont pas été pris en compte pour le calcul de la moyenne des compositions et la définition des caractéristiques de l'atelier. les compositions chimiques de l'atelier de la Sarra présentent de nombreuses variations et l'echantillonnage analysé ne correspond qu'à une petite partic de la distribution. On donnera a titre indicatif les moyemes des teneurs des 19 exemplaires dont les compositions se ressemblent le plus (tabl. VII).

Afin de compléter l'étude des compositions des céramiques communes sombres de l'atelier de la Sarra, nous avons écé amenées à analyser huit exemplaires de céramiques communes sombres découvertes sur le site de Trion peu éloigné du site de la Sarra. Ia question se posait de savoir si les céramiques découvertes sur ce site, et qui pourraient être des productions locales, avaient 
Tabl. VII. composition chimique moyenne des céramiques communes sombres de l'atelier de la Sarra $(n=19)$.

Éléments majeurs en pourcentages d'oxydes

\begin{tabular}{|c|c|c|c|c|c|c|c|c|c|c|}
\hline $\begin{array}{l}\text { La Sarra } \\
\text { pâte I } \\
(\mathrm{n}=19)\end{array}$ & $\mathrm{Na}_{2} \mathrm{O}$ & $\mathrm{K}_{2} \mathrm{O}$ & $\mathrm{MgO}$ & $\mathrm{CaO}$ & $\mathrm{MnO}$ & $\mathrm{Al}_{2} \mathrm{O}_{3}$ & $\mathrm{Fe}_{2} \mathrm{O}_{3}$ & $\mathrm{SiO}_{2}$ & $\mathrm{TiO}_{2}$ & 2 \\
\hline $\mathrm{m}$ & 0,28 & 1 & 0,6 & 1,8 & 0,032 & 16,8 & 5,9 & 72,2 & 0,83 & 0,43 \\
\hline$\sigma$ & 0,04 & 0,1 & 0,1 & 0,2 & 0,015 & 0,7 & 0,2 & 0,5 & 0,01 & 0,11 \\
\hline$\sigma \%$ & 16 & 7 & 6 & 13 & 29 & 4 & 3 & 1 & 2 & 25 \\
\hline
\end{tabular}

Éléments traces en PPM

\begin{tabular}{|c|r|c|c|r|r|r|r|r|r|r|}
\hline $\begin{array}{c}\text { La Sarra } \\
\text { pâte I } \\
(\mathbf{n}=19)\end{array}$ & $\mathbf{R b}$ & Sr & Ba & Ni & Zn & Cr & Zr & La & Ce & V \\
\hline $\mathbf{m}$ & 45 & 96 & 455 & 68 & 64 & 78 & 313 & 68 & 130 & 93 \\
\hline$\sigma$ & 6 & 11 & 125 & 6 & 7 & 3 & 17 & 17 & 15 & 5 \\
\hline$\sigma \%$ & 14 & 11 & 27 & 9 & 10 & 3 & 5 & 25 & 12 & 6 \\
\hline
\end{tabular}

des compositions identiques ou proches de celles de l'atelier de la Sarra.

On remarque qu'aucun des exemplaires provenant du site de Trion ne se classe parmi les exemplaires de la Sarra, de plus ils ne ressemblent pas non plus aux exemplaires marginaux de la Sarra. Les compositions sont assez variées et seuls quatre excmplaires ont des compositions comparables. Ceci associé au fait que macroscopiquement il est possible de distinguer au moins deux groupes de pâte parmi ce matériel ne permet pas de proposer une composition chimique moyenne des productions de cet atelier.

Les exemplaires provenant de Trion correspondent probablement aux productions d'un atelier différent de celui de la Sarra.

Pour expliquer la grande variété de groupes de pâte observés macroscopiquement et la dispersion des compositions chimiques dans les ateliers de la Sarra et de Trion, on peut proposer deux hypothèses. Soit il existe à Lyon plusicurs ateliers de céramiques communes sombres à pâte grossière (ce qui est très probable), auquel cas les divers groupes observés correspondraient aux productions de divers ateliers, soit les diverses compositions chimiques correspondent à des variations locales au sein de la formation argileuse et l'utilisation de gisements limités a conduit à des variations d'approvisionnement au sein d'un même atelier. Ces deux phénomènes peuvent d'ailleurs intervenir conjointement.

A. $S$. 